\title{
A CSI ADAPTIVE OPTIMAL RELAY NETWORK DESIGN BASED ON PN-SNR FOR MIMO NETWORKS
}

\author{
Gautham.R ${ }^{1}$, Electa Alice ${ }^{2}$ \\ ${ }^{I} M$-Tech Student, Electronics and Communication Department, TJIT, Karnataka, India \\ ${ }^{2}$ Assistant Professor, Electronics and Communication Department, TJIT, Karnataka, India
}

\begin{abstract}
In this work, an effective measure known as the got sign to clamor proportion (SNR) per unit power in the increase and forward hand-off system is received. This technique is known as the Power standardized SNR likewise called as PN-SNR. In a numerous Relay arrange the PN-SNR amplification and to consider the system execution. Firstly for a solitary transfer arrange the perfect hand-off force control conspire that increase the PN-SNR for the given transmitter force is figured. At that point for the Multi transfer system with an aggregate hand-off force imperative of the amplified PN-SNR is gotten, a most extreme quality can be inferred by this a widespread ideal arrangement is acquired by the inclination rising calculation strategy. In the numerous handoff system with the different force requirement on the every transfer a calculation is proposed to get the general ideal arrangement furthermore it is a base unpredictability calculation for an imperfect arrangement.
\end{abstract}

Key words: Relay Network, PN-SNR, Efficiency, Power Control, Outage Probability $* * *$

\section{INTRODUCTION}

The interest for the Wireless systems is frequently expanding interest for the higher information rates without trading off with the validity. The most ideal approach to enhance the validity in the information rate and/or is to utilize the helpful plans in the system. The force control and system streamlining are the points in the hand-off system plans .There have been a great deal number of execution parameters, for example, sign to clamor ratio(SNR) boost ,Throughput expansion and mistake rate minimization for a consistent transmit power. As the interest for the remote clients and the remote activity quickly expands the expansion in the asset usage in the remote framework additionally builds prompting the green house impact and the corruption of the eco framework. Thus eco inviting correspondence plan has critical significance in the up and coming years. The reasonable proficiency measures incorporate otherworldly productivity and vitality effectiveness.

Recognizing with the phantom productivity metric, the PNSNR is a more characteristic proficiency measure the execution. Distinguishing with the vitality effectiveness metric.PN-SNR will not hang the network in very minimum power systems.

\section{OBJECTIVE OF THE PROPOSED WORK}

The goal of the proposed work is to fuse a novel productivity measure, in particular the got signal-tocommotion proportion per unit power in amplify and forward strategy. The metric is characterized as force standardized SNR (PN-SNR). To addresses the previously stated constraints of ghastly productivity and vitality proficiency has prompted the investigation of the new execution measure specifically SNR per unit power or PNSNR, to outline network. For a solitary client arrange the PN-SNR is characterized.

$$
\eta=\frac{S N R}{P_{\text {Total }}}
$$

Where SNR is between two end points i.e Tx and Rx and Ptotal is the force devoured in the circuit. The factor speaks to the possible got SNR per unit transmit power. If the received commotion has a minimal difference, can similarly be seen as the propagator SNR.

\section{LITERATURE SURVEY}

\section{1 "User Cooperation diversity: System Descryption}

The utilization of some sort of assorted qualities. In this two-section paper, we propose another type of spatial assorted qualities, in which differing qualities additions are accomplished by means of participation of portable clients information rate and nature of administration are restricted by the way that, inside the length of any given call, they encounter extreme verities in sign lessening, in this manner reauiring

\subsection{Client Collaboration Assorted Qualities}

This is the second of a two-section paper on another type of spatial differing qualities, where assorted qualities increases are accomplished through the collaboration of versatile 
clients. Part I portrayed the client collaboration idea and proposed a participation technique for a customary codedivision different access (CDMA) framework. Part II examines the participation idea further and considers down to earth issues identified with its usage. Specifically, we explore the ideal and imperfect collector outline, and present execution examination for the customary CDMA usage proposed in Part I.

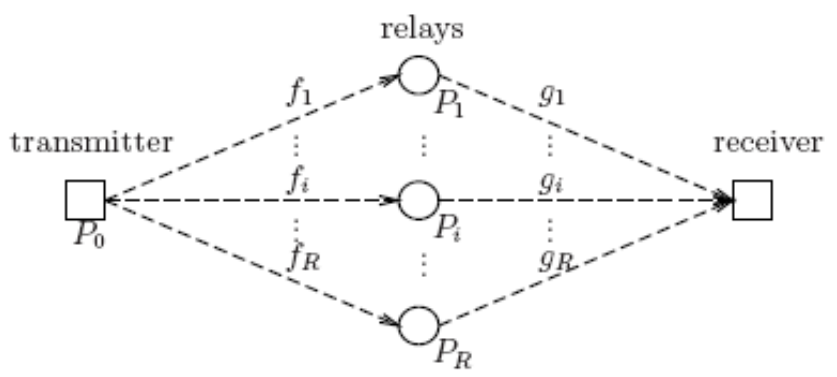

Fig 1. Two Hop Relay Network

\section{RELAY NETWORKS}

A hand-off framework is a far reaching class of framework circuit routinely used as a piece of remote frameworks, where the Tx and Rx are coupled by strategy for a couple of center points. In such a framework the Tx and Rx can't pass on to each other particularly in light of the way that the detachment between the Tx and Rx is more paramount than the transmission extent of them two, thusly the prerequisite for transitional node(s) to exchange..

The least complex transferring system comprises of three hubs, specifically source, destination, and a third hub supporting the immediate correspondence amongst source and destination meant as hand-off. In the event that the immediate transmission of a message from source to destination is not (completely) effective, the caught data from the source is sent by the hand-off to achieve the destination through an alternate way.

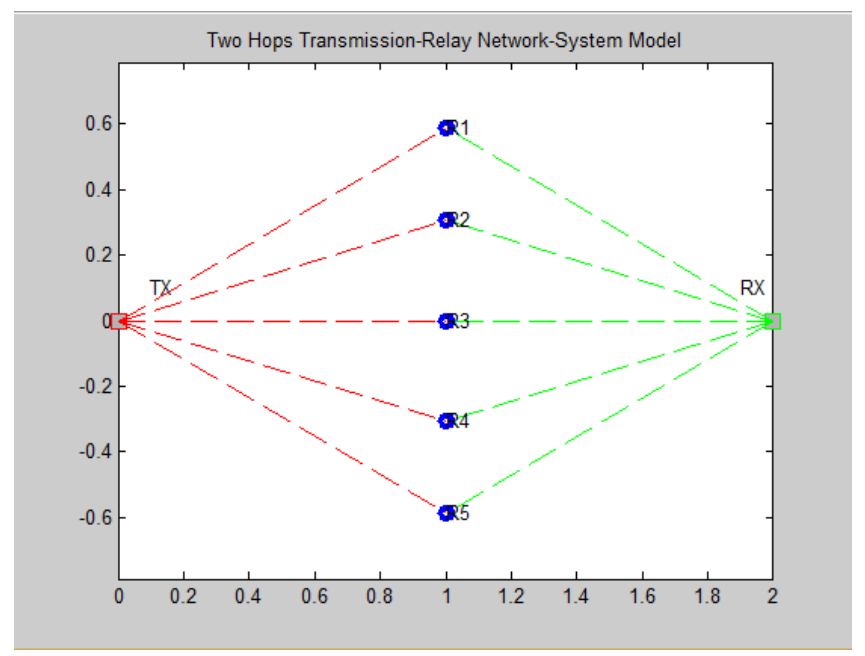

Fig 2. Two Hop Relay Transmission Network

\section{RESULTS}

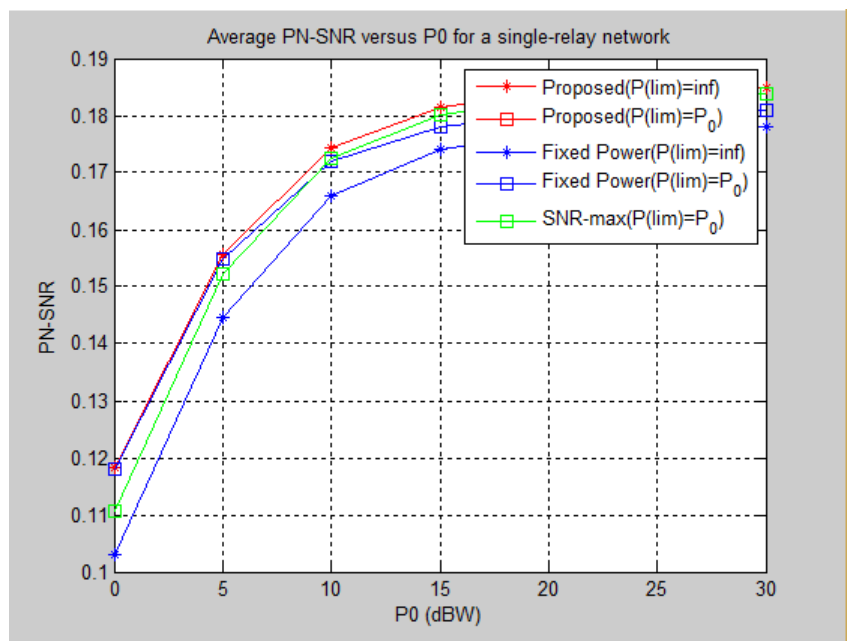

Fig 3. Average PN-SNR Vs P0 for a single relay

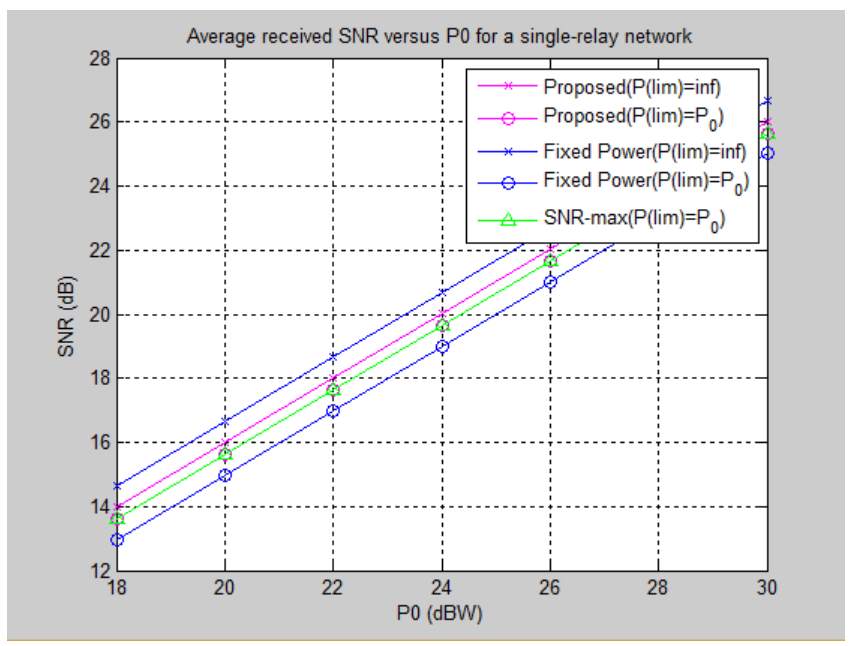

Fig 4. Average received SNR Vs P0 for single relay

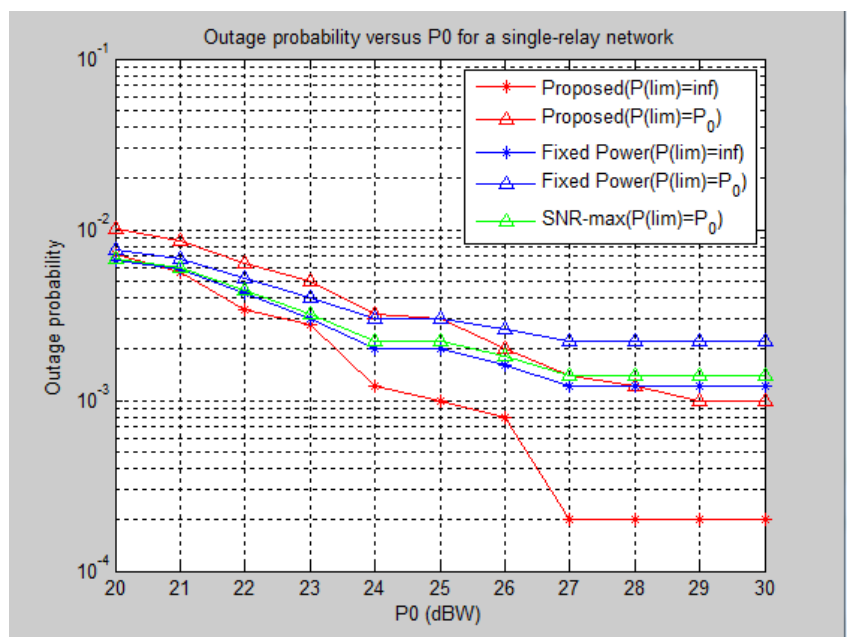

Fig 5. Outage Probability Vs P0 for single relay

\section{Average PN-SNR Vs P0 for a single relay}

In the average PN-SNR Vs P0 we can verifiy thet the power distribution and the power consumed in the proposed method is less than that of the existing method. 


\section{Average received SNR Vs P0}

By fixing the constant power in the power limiting case we can come to a conclusion that by maximizing the SNR the information travels to a longer distance with the less power consumption.

\section{Outage Probability Vs P0 for single relay}

The outage probability shows that how efficiently the system can work in the critical conditions by the above system we can prove that more the samples less the outage. This shows the minimum outage for the proposed method.

\section{CONCLUSIONS}

In this paper we received another parameter called Power Normalized SNR (PN-SNR) to plan a productive hand off system. Performance of the proposed plans is broke down and contrasted and the current plans. Compared with the SNR boosting outline and the PN-SNR altogether has higher effectiveness and decrease the blackout likelihood there by bringing out the proficient use of force and maintaining the strategic distance from the system trap when force approaches zero.

\section{REFERENCES}

[1] A. Sendonaris, E. Erkip, and B. Aazhang, "User cooperation diversity - part I: system description," IEEE Trans. Commun., vol. 51, no. 11, pp. 1927-1938, Nov. 2003.

[2] "User cooperation diversity-part II: implementation aspects and performance analysis," IEEE Trans. Commun., vol. 51, no. 11, pp. 1939-1948, Nov. 2003.

[3] J. N. Laneman, D. N. C. Tse, and G.W.Wornell, "Cooperative diversity in wireless networks: efficient protocols and outage behavior," IEEE Trans. Inf. Theory, vol. 50, no. 12, pp. 3062-3080, Dec. 2004.

[4] Y. Jing and H. Jafarkhani, "Network beamforming using relays with perfect channel information," IEEE Trans. Inf. Theory, vol. 55, no. 6, pp. 2499-2517, June 2009.

[5] Y. Zhao, R. Adve, and T. J. Lim, "Improving amplifyand-forward relay networks: optimal power allocation versus selection," IEEE Trans. Wireless Commun., vol. 6, no. 8, pp. 3114-3123, Aug. 2007.

[6] T. M. Cover and A. E. Gamal, "Capacity theorems for the relay channel," IEEE Trans. Inf. Theory, vol. 25, no. 5, pp. 572584 , Sept. 1979

[7] M. Dohler and Y. Li, Cooperative Communications: Hardware, Channel and PHY. John Wiley \& Sons, 2010.

[8] C. J. K. Y. W. P. Hong, W. Huang, Cooperative Communications and Networking: Technologies and System Design. Springer, 2010.

[9] P. Larsson, "Large-scale cooperative relaying network with optimal combining under aggregate relay power constraint," in Proc. 2003 Future Telecommunications Conference, pp. 160-170.

[10] Y. Jing and H. Jafarkhani, "Network beamforming using relays with perfect channel information," IEEE Trans. Inf. Theory, vol. 55, no. 6, pp. 2499-2517, June 2009

\section{BIOGRAPHIES}

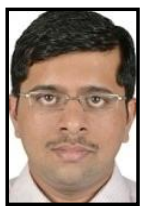

Mr Gautham.R is studying M.Tech at T John Institute of Technology and is doing the project based on "CSI ADAPTIVE OPTIMAL RELAY NETWORK BASED ON PN-SNR FOR MIMO NETWOR"

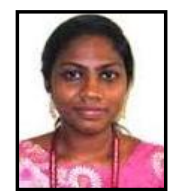

Ms Electa Alice Jayarani is currently working as a Assistant Professor in TJIT and is pursuing her PH.D in Audio Water Marking. 